\title{
An Improved Region Contrast and Global Distribution Saliency Detection algorithm
}

\author{
Xiaohong Hao ${ }^{1, a}$, Yifang Yuan ${ }^{1, b}$ and Wanfei Jiang ${ }^{1, c}$ \\ ${ }^{1}$ Langongping road of No. 287, Qilihe district, Lanzhou, China \\ a316475958@qq.com, b1987701140@qq.com, '136172464@qq.com
}

\begin{abstract}
Keywords: Saliency detection, region contrast, global distribution
Abstract. According to the local contrast and global distribution of an image, this paper detecting salient images through bottom-up data driven . First, this paper using adaptive segmentation method divided image into non-overlapping images, improved Block and Chessboar distance from a linear combination to replace the Euclidean distance method to calculate the regional features of contrast functions, then calculate the global distribution of feature functions, finally fusion of the above features for computing saliency map. The algorithm taking into account local features and global features to get more accurate saliency map. Test our method on the international public data sets MSRA-1000,the experimental result proves that the images extracted by this method are more accurate and more clearly, while reducing the calculation time of regional characteristics, having strong noise and high texture regions resistance, and can basically ignore the complex background.
\end{abstract}

\section{Introduction}

An important feature of human vision is assigned limited processing resources to the interest area on the priority. Detection of significant regional study is to simulate human visual attention mechanisms to extract the higher significant region from the image. Significant area detection is widely used in image segmentation, image compression, image retrieval, content-aware, target identification and other fields.

The methods of detecting saliency image can be divided into two types: one is Bottom-up [1]data-driven, the other is Top-down task-driving. This paper is focus on detecting an image's saliency region by Bottom-up method. Bottom-up data-driven model is divided into two main categories: one method using local features [2] of the image, and the other using global features of the image. Individually saliency detection algorithm based on global features of the image, when the image has some complicated main targets and some markedly different regions, it will be easy to miss the main targets in the course of processing. The accuracy and speed of the feature detection by Separate global and local feature still have further room for improvement, and still have the problems of the fuzzy profiles for saliency regional , and poor noise and high texture resistance ability for noise and high texture. Above the issue, this paper propose an improved region contrast and statistics saliency detection.

\section{SLIC Algorithm for Image Segmentation.}

This paper uses SLIC(simple linear iterative clustering) [3] based on the similarity of the color and distance. Super pixel divided by converting color images to CIELAB color space and 5-dimensional feature vectors of XY coordinates, then the 5-dimensional feature vectors constructed metric, local clustering of pixels of the image. The segmented images as shown in Fig.1

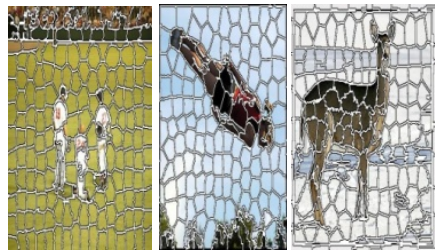

Fig. 1 The results of SLIC image segmentation 


\section{Improving Local Contrast Feature Function.}

Regional Feature of Contrast Function. This paper inspired from papers [4] based on regional contrast method, The same is when calculated the contrast between two regions, we use regional spatial distance as a weight of regional contrast, combined with the feature of the color contrast composition of image region features function, making the far region have a smaller contribution, and the near region have a bigger contribution to image contrast. Unlike the Euclidean distance to calculate the two regions, we use the linear combinations of Blocks and Chessboard distance instead of the Euclidean distance. The formula is as follows:

$$
\begin{aligned}
& C_{k}=\sum_{\substack{m=1 \\
m \neq k}}^{200} D_{r}\left(r_{k}, r_{m}\right) \\
& D_{r}\left(r_{k}, r_{m}\right)=\sum_{n_{k}}^{n_{m}} \frac{p_{k}(i) \cdot p_{m}(j) \cdot\left\|r_{k}(i)-r_{m}(j)\right\|_{2}}{1+d_{r}\left(r_{k}, r_{m}\right)} \\
& d_{r}\left(r_{k}, r_{m}\right)=\lambda L_{1}+\theta L_{2} \\
& =\lambda\left(\left|\mathrm{x}_{k}-x_{m}\right|+\left|y_{k}-y_{m}\right|\right)+\theta \max \left(\left|\mathrm{x}_{k}-x_{m}\right|,\left|y_{k}-y_{m}\right|\right) \\
& D_{r}\left(r_{k}, r_{m}\right)=\sum_{n_{k}}^{n_{m}} \frac{p_{k}(i) \cdot p_{m}(j) \cdot\left\|r_{k}(i)-r_{m}(j)\right\|_{2}}{1+\lambda\left(\left|\mathrm{x}_{k}-x_{m}\right|+\left|y_{k}-y_{m}\right|\right)+\theta \max \left(\left|\mathrm{x}_{k}-x_{m}\right|,\left|y_{k}-y_{m}\right|\right)}
\end{aligned}
$$

$C_{k}$ is The contrast of the k-th regional $r_{k}$ feature function; $r_{k}, r_{m}$ representing the image of the kth and the m-th region; $d\left(r_{k}, r_{m}\right)$ is the Euclidean distance of $r_{k}, r_{m} ;\left(x_{k}, y_{k}\right),\left(x_{m}, y_{m}\right)$ representing the centroid of $r_{k}, r_{m} ; L_{1}, L_{2}$ representing the Blocks and Chessboard distance of $r_{k}, r_{m} ; n_{k}, n_{m}$ representing $r_{k}, r_{m}$ contains the total number of colors; $p_{k}(i)$ representing the probability of $r_{k}(i)$ in region $r_{k} ; p_{m}(j)$ representing the probability of $r_{m}(j)$ in $r_{m}$;From the foregoing, Euclidean distance require once addition operation of square root and the twice square to be calculated; but the Blocks and Chessboard distance we use don't require to calculate the addition operation of square root and the square operation. Calculating $D_{r}\left(r_{k}, r_{m}\right)$ can save $n_{m} \times n_{k}$ times calculation of the addition operation of square root and $2 n_{m} \times n_{k}$ times of squared operation .In this paper we value $\lambda=0.004, \theta=0.2$.

Spatial Position of the Feature Function. Using the distance relationship between regions and image center, constructed the spatial position of the feature function. So from the center of the image area near significantly high, far from the center opposite a significant low. The spatial position of feature function of normalized area of the image is defined as follows :

$$
P_{i}=1-\sqrt{\left(\frac{x_{i}}{h}-\frac{1}{2}\right)^{2}+\left(\frac{y_{i}}{w}-\frac{1}{2}\right)^{2}}
$$

$P_{i}$ is The position feature function of the $\mathrm{i}$-th region $r_{i}, P_{i} \in[0,1] ;\left(x_{i}, y_{i}\right)$ is the centroid coordinates of the $\mathrm{i}$-th region $r_{i} ; h, w$ is The height and width of the whole image.

Salient Regions Detection. Salient region function defined as follows

$$
S_{1}\left(r_{i}\right)=P_{i} \cdot W_{i} \cdot \exp \left(\delta C_{i}\right)
$$

$S_{1}\left(r_{i}\right)$ is the salient value of the $\mathrm{i}$-th region ; $\delta$ is the results of the image contrast ratio enhancement experiment result, it shows that when $=1.6$, we can get better experiment result. 


\section{Calculation of Global Distribution of Feature Function}

According to the principles that regional saliency and the spatial distribution of concentration of regional is proportional, spatial distribution of the smaller variance, significantly higher. Specific to the region, the spatial variance of each region is smaller, significantly higher in the region, whereas areas of significantly lower. Based on the principle significant value is inversely proportional to the variance of the distribution, calculating region significant values, the formula is as follows:

$$
\begin{aligned}
& S_{2}\left(r_{i}\right)=\log \left(\sqrt{1 / D_{\left(r_{i}\right)}}\right) \\
& g\left(C_{r_{i}}, C_{r_{j}}\right)=\frac{1}{Z_{x}} \exp \left(\frac{-\left\|C_{r_{i}}-C_{r_{j}}\right\|^{2}}{\sigma_{x}{ }^{2}}\right) \\
& D\left(r_{i}\right)=\sum_{j=1}^{n} g\left(C_{r_{i}}, C_{r_{j}}\right) \cdot d\left(x_{i}, y_{i} ; x_{j}, y_{j}\right)
\end{aligned}
$$

$D\left(r_{i}\right)$ is the Spatial distribution of regional variance; $g\left(C_{r_{i}}, C_{r_{j}}\right)$ is the similar color values of the the $r_{i}, r_{j}$ region weight; $C_{r_{i}}, C_{r_{j}}$ representing the corresponding color value of region $r_{i}, r_{j}$; $d\left(x_{i}, y_{i} ; x_{j}, y_{j}\right)$ is the centroid coordinates of Euclidean distance of region $r_{i}, r_{j} ;\left(x_{i}, y_{j}\right),\left(x_{j}, y_{j}\right)$ representing the regional center of centroid coordinates $; Z_{x}$ is the the normalization factor; $\sigma_{x}^{2}=2$.

\section{Ultimate feature function}

Using Gaussian model combined the two test results in different weights, then caculate the salient value of each region. Formulas are as follows:

$$
S\left(r_{i}\right)=\exp \left(\alpha \cdot S_{1}\left(r_{i}\right)+\partial \cdot S_{2}\left(r_{i}\right)\right)
$$

$\alpha, \partial$ are weight control parameters represent two ways of detecting salient impact on the final result.In this paper we chose $(\alpha=1, \partial=2)$, then $F_{\beta}$ can reach the highest value.

\section{Simulation and analysis}

In order to verify the validity of the algorithm in this paper, we use the international public Dataset MSRA-1000 test our method, running in a matlab2014 environment. Compared our method with the current popular GB, SR, and AC, CA, IT and MZ methods, The saliency map and comparison shown in Fig.2.

In order to fully assess the accuracy of this method, using precision, recall and $F_{\beta}$ to compare. We get comparative test results as shown in Fig.3, 4 and Table1. Because this paper use a linear combination of Block and Chessboard distance replacing the Euclidean distance method to calculate the regional characteristics of contrast functions, so improved overall speed. In addition, our method having strong noise and high texture regions Resistance, and can basically ignore the complex background. $F_{\beta}$ defined as follows:

$$
F_{\beta}=\frac{(1+\beta) \cdot \text { Pr ecision } \cdot \text { Re call }}{\beta^{2} \cdot \text { Pr ecision }+ \text { Re call }}
$$

The formula references[5], and $\beta^{2}=2$. 


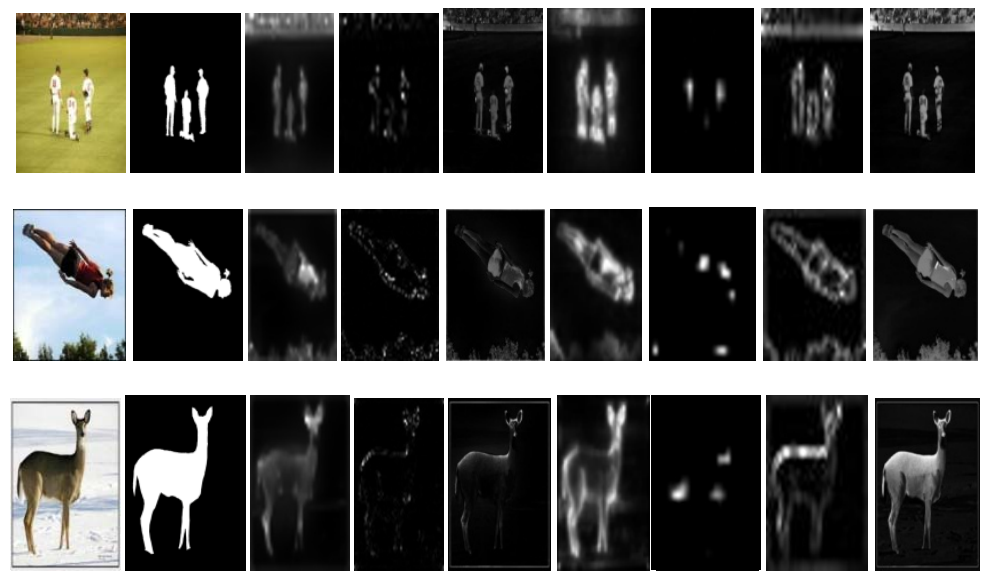

Original GT GB SR AC CA IT MZ Ours

Fig. 2 The comparison of salient region detection methods

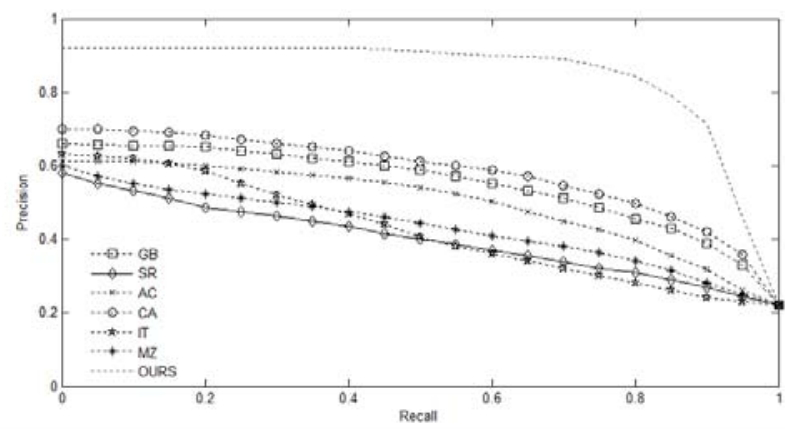

Fig.3 Precision-recall curves of other methods

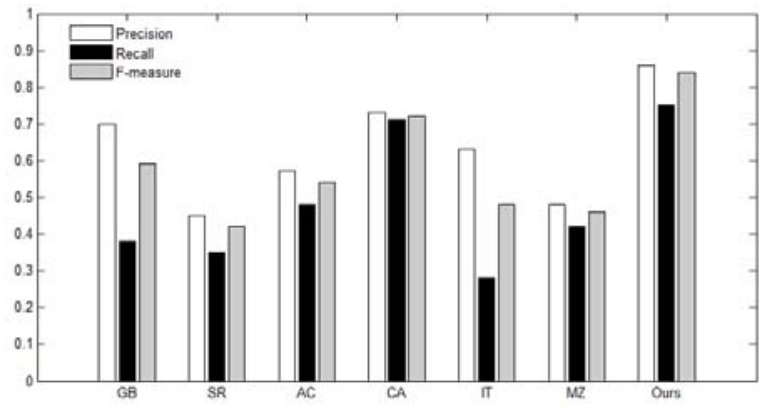

Fig.4 Precision, recall,F-measure

\begin{tabular}{cccccccc}
\multicolumn{7}{c}{ Table 1 } & \multicolumn{6}{c}{ Comparison of average running time } \\
\hline & GB & SR & AC & CA & IT & MZ & Ours \\
\hline Times & 2.097 & 0.034 & 0.159 & 53 & 0.842 & 0.03 \\
0.143 & & & & & & & \\
Code & Matlab & Matlab & Matlab & Matlab Matlab & Matlab & Matlab \\
\hline
\end{tabular}

\section{Conclusion}

This paper improve a method of the local contrast and global distribution to detect the saliency region. having strong noise and high texture regions resistance. But we also have some limitations, to the similar background color of the object, test results have not been satisfactory. In future work, we hoped that this method was improved, and will have further consideration of the specific target information, and detect a better effect significant area , and make it more widely available.

\section{Reference:}

[1] ITTI L,KOCH C,NIEBUR E.A model of saliency-based visual attention for rapid scene analysis[J].IEEE Transaction on Pattern Analysis and Machine Intelligence,1998,20(11):1254-1259 [2] Harel J, Graph-based visual saliency. Proceedings of the 2006 MIT Annual Conference on Neural Information Processing Systems. Vancouver, Canada: The MIT Press, 2006. 545-552

[3] ACHANTA R, et al. SLIC superpixels compared to state-of-the-art superpixel methods [J] . IEEE Trans on Pattern Analysis and Machine Intelligence, 2012, 34 ( 11 ) :2274-2282.

[4] Wenjie Zhang, Qingyu Xiong. Image salient region detection algorithm based on contrast and spatial location[J]Journal of Jilin University(Engineering and Technology Edition)2015, 45(5)

[5] ACHANTA R, HEMAMI S,ESTRADA F. Frequency-tuned salient region detection [C].CVPR,2009:1597-1604 